\title{
Thirty Joint Russian-German Meetings on ECRH and Gyrotrons - A Retrospect -
}

\author{
Manfred Thumm ${ }^{1,2}$, \\ ${ }^{1}$ Institute for Pulsed Power and Microwave Technology (IHM), \\ ${ }^{2}$ Institute of Radio Frequency Engineering and Electronics (IHE), \\ Karlsruhe Institute of Technology (KIT), 76131 Karlsruhe, Germany, manfred.thumm@kit.edu
}

\section{Foundation of Russian-German Cooperation on ECRH and Gyrotrons}

The USSR-FRG scientific-technical cooperation in the field of electron cyclotron resonance heating (ECRH) and gyrotrons was founded in 1988, when delegations of the German Ministry for Research and Technology (BMFT) (Dr. S. von Krosik) and of the USSR State Committee for Utilization of Atomic Energy (Dr. M.P. Moiseeva) met at the I.V. Kurchatov Institute of Atomic Energy (KIAE) in Moscow to establish a cooperation in the area of thermonuclear fusion. Leading scientific members of the German delegation were Prof. R. Wienecke (Institute for Plasma Research (IPF), University of Stuttgart) and Dr. G. Grieger (Max Planck Institute for Plasma Physics (IPP), Garching). The USSR delegation was represented by Dr. B.B. Kadomtsev, Dr. G.A. Eliseev and Dr. W.S. Strelkov (KIAE) and Dr. V.A. Flyagin (Institute of Applied Physics (IAP) of the Soviet Academy of Sciences, Gorky). In a letter of Dr. Flyagin to Prof. Wienecke dated June 6, 1988, V.A. Flyagin gave the information, that "in the future we shall be able to organize seminars in the USSR".

\section{The First Meetings in 1989 and 1990}

The topics of the first USSR-FRG Meeting on ECRH and Gyrotrons were discussed during a scientific stay of Dr. G.S. Nusinovich at Kernforschungszentrum Karlsruhe (KfK) in July 1988. The preliminary agenda of the workshop was:

-Status reports and future plans on ECRH system development and on ECRH experiments -Special subjects of gyrotron development

-Design of Magnetron Injection Guns (MIGs)

-Whispering gallery vs. circular mode gyrotrons

-Dielectric vacuum windows, material and design

-Gyrotron operation experience

-Alternative millimeter ( $\mathrm{mm})$-wave sources

-Advances in electrodynamic systems

-Development perspectives of transmission lines -Quasi-optical (q.o.) vs. oversized waveguides.

The first meeting took place from March 6-10, 1989 in Germany at KfK Karlsruhe, IPF Stuttgart and IPP Garching. The USSR delegation had 5 members: G.G. Denisov, V.A. Flyagin, G.S. Nusinovich, M.I. Petelin from IAP Gorky and A.A. Borschegovsky from KIAE Moscow. The approximately 20 German participants came from KfK Karlsruhe, IPF Stuttgart, IPP Garching, RWTH Aachen and Technical University of Hamburg-Harburg. The meeting was accompanied by laboratory visits at KfK, IPF and IPP.

Since 1989 this series of annual meetings is alternatively organized in Germany and Russia. The second meeting was held at KIAE Moscow and IAP Gorky. The participating institutions were: KIAE Moscow, Institute of General Physics Moscow, IAP Gorky, IPP Garching, IPF Stuttgart and KfK Karlsruhe. The FRG delegation consisted of five members: V. Erckmann from IPP, W. Kasparek and M. Thumm from IPF and G. Dammertz and H.-U. Nickel from KfK. The total number of participants was about 50. 22 reports were presented and discussed. Technology items were: long-pulse $140 \mathrm{GHz}$ gyrotrons, cryogenically cooled windows, combined q.o. and waveguide transmission lines. In the field of plasma physics, T-10 and W7-AS ECRH and electron cyclotron current drive (ECCD) experiments were discussed. Visits were organized to the tokamaks $\mathrm{T}-10$ and $\mathrm{T}-15$ at KIAE and to the laboratories involved in the development of gyrotrons, relativistic microwave devices, electrodynamic systems and plasma physics at IAP. In 1991, the city of Gorky was re-named as Nizhny Novgorod.

\section{Agreement on Cooperation between IAP and IPP}

During the $3^{\text {rd }}$ meeting of this workshop series from June 12-18, 1991 at Karlsruhe, Stuttgart and Garching in Germany, the Russian delegation reported about gyrotrons, CARMs, electrodynamic systems, and ECRH\&CD on T-10, whereas the German team presented results on transmission lines, windows, electrodynamic systems, and ECRH\&CD on the stellarator W7-AS. On course of this meeting, on June 18, 1991, an Agreement of Cooperation between IAP and IPP was signed aiming at the use of $0.5 \mathrm{MW}$, $140 \mathrm{GHz}$ Soviet gyrotrons with $0.5 \mathrm{~s}$ pulse duration at W7-AS and ASDEX Upgrade. The two parties of the Agreement decided that the first stage of the cooperation should cover in particular the following fields:

-Test of Soviet high power gyrotrons at the W7-AS stellarator with respect to frequency stability, mode purity and power control (in collaboration with KfK and KIAE);

- Tests of the q.o. transmission line developed by IPF with respect to power capability, losses and transmission efficiency (in collaboration with IPF);

-Tests of microwave barrier windows under high power conditions;

-Plasma physics experiments (in collaboration with $\mathrm{KIAE}, \mathrm{IPF}$ and $\mathrm{KfK}$ ): plasma generation and $2^{\text {nd }}$ harmonic X-mode heating in W7-AS at $140 \mathrm{GHz}$, ECCD, on-and off-axis heat-wave experiments and combined ECRH and NBI.

Later, this cooperation led to the delivery of commercial 140 and 140/105 GHz GYCOM gyrotrons to W7-AS and ASDEX Upgrade. 
Scientific and Technological Highlights of the Thirty Joint Russian-German Meetings

A series of novel developments have been achieved during this Russian-German cooperation.

\section{Plasma Physics}

ECRH\&CD experiments have been performed in the tokamaks $\mathrm{T}-10$ and ASDEX-Upgrade, in the stellarators W7-AS and W7-X, and in the gas dynamic mirror trap (GDT), leading to substantial progress in understanding the physics of fusion plasmas.

T-10: $1^{\text {st }}$ and $2^{\text {nd }}$ harmonic ECRH\&CD experiments with power levels up to $3 \mathrm{MW}$; ECCD with reversed magnetic shear; demonstration of internal transport barrier formation by high-efficiency off-axis ECRH, ECRH\&CD experiments on density pump-out; heavyion impurity control and influence of plasma reflections on gyrotron operation.

ASDEX-Upgrade: $2^{\text {nd }}$ harmonic ECRH\&CD experiments; complete stabilization of (3/2) and (2/1) neoclassical tearing modes (NTMs) by ECCD, record temperatures $\left(T_{e}, T_{i}>13 \mathrm{keV}\right)$ with ECCD sustained thermal transport barrier; electron heat transport (steady state and transient phase) explained by iontemperature-gradient (ITG) / trapped-electron-mode (TEM) turbulence after stabilization of (3/2)-NTM at $\beta_{\mathrm{N}}=3.1$; consistent experimental and theoretical explanation of flattened density profiles (pump out) under intense ECRH; first operation of industrial $1 \mathrm{MW}, 10 \mathrm{~s}$ two-frequency gyrotrons; H-mode discharges with ITER-relevant safety factor using $3^{\text {rd }}$ harmonic ECRH; 4.4 MW ECRH\&CD using 8 gyrotrons for ELM mitigation and NTM stabilization; successful operation of a compact fast-directional switch (FADIS) in the ECRH transmission system; advanced tokamak experiments in W-coated ASDEXUpgrade and non-inductive $\mathrm{H}$-mode operation with safety factor- $(95)=5.3$; excellent performance of new two-frequency gyrotrons for the ECRH-3 system.

W7-AS: Discovery of $\mathrm{H}$-mode discharges using $70 \mathrm{GHz} 1^{\text {st }}$ harmonic O-mode and $140 \mathrm{GHz} 2^{\text {nd }}$ harmonic X-mode ECRH; installation of Collective Thomson Scattering (CTS) diagnostics and first results on CTS ion temperature measurements; investigation of $\mathrm{O} 2$-mode heating and mode conversion schemes; first experiments on $\mathrm{O}-\mathrm{X}-\mathrm{B}$ conversion heating, plasma heating and highly efficient current drive via Bernstein waves in over-dense plasmas $\left(>3 \times 10^{20} / \mathrm{m}^{3}\right)$, achievement of $7 \mathrm{keV}$ electron temperature with 2.7 MW ECRH; introduction of electron Bernstein wave emission diagnostics; extreme power densities in ECCD experiments and development of related non-linear theoretical description.

W7-X: Plasmas generated and heated by X2-mode ECRH only; parallel operation of ten $1 \mathrm{MW}, 140 \mathrm{GHz}$ gyrotrons and a unique purely q.o. transmission line; successful demonstration of O2-mode operation above the X2-cutoff at plasma densities up to $1.4 \times 10^{20} / \mathrm{m}^{3}$, in combination with pellet injection a stellarator record triple product of $0.66 \times 10^{20} \mathrm{~m}^{-3} \mathrm{~s} \mathrm{keV}$ and $T_{e}=T_{i}=3.9 \mathrm{keV}$ were achieved; successful first operation of two remote steering launchers at; commissioning and first results of the CTS diagnostic. GDT: $0.8 \mathrm{MW}, 54 \mathrm{GHz} \mathrm{ECH}$ doubles the neutron rate.

\section{Gyrotrons and ECRH\&CD Technology}

Gyrotron, transmission line and ECRH\&CD technology development has been done at IAP, IPF (now IGVP), IPP and KfK (later FZK and now KIT).

Gyrotrons: Introduction of single-stage depressed gyrotron collector (SDC) and additional $50 \mathrm{~Hz}$ transverse electron-beam sweeping; demonstration of $70 \%$ efficiency and $99 \%$ output-mode purity of an industrial $75 \mathrm{GHz}$ gyrotron; $0.2 \mathrm{MW}, 84 \mathrm{GHz}$, continuous wave $(\mathrm{CW})$ gyrotron for the LHD stellarator at NIFS; megawatt-class $110 \mathrm{GHz}$ gyrotron for TEXTOR (pulse length $2 \mathrm{~s}$ ), $140 \mathrm{GHz}$ gyrotrons for T-10, ASDEX-Upgrade (10 s), W7-AS (3 s) and W7-X (1800 s) and $170 \mathrm{GHz}$ gyrotrons for ITER $(1000 \mathrm{~s}, 1 \mathrm{kHz}$ power modulation); $1.2 \mathrm{MW}, 100 \mathrm{~s}$, $52 \%$ efficiency operation of the Russian ITER tube and corresponding 1.5-2 MW development for ITER Upgrade; development of short-pulse, coaxial-cavity gyrotrons: $1.5 \mathrm{MW}, 140 \mathrm{GHz}$ with dual-beam output coupler and two windows, $2.2 \mathrm{MW}, 165 \mathrm{GHz}$ with 49 $\%$ efficiency at $2 \mathrm{MW}$ (with SDC), $2.3 \mathrm{MW}, 170 \mathrm{GHz}$ with $46 \%$ efficiency at $2.2 \mathrm{MW}$ (with SDC); first short-pulse 114-162 GHz, 1 MW gyrotrons with stepfrequency tuning using Brewster output windows and experiments on fast step-tuning using a hybrid gyrotron magnet; $1 \mathrm{MW}$ two-frequency and multifrequency gyrotrons; future DEMO gyrotrons and the development of other types of mm- and sub-mm-wave sources for non-fusion applications were discussed.

ECRH\&CD Technology: Introduction, test and application of Chemical Vapor Deposition (CVD) diamond and doped silicon as high-power window materials; due to the superior mechanical characteristics, synthetic diamond is the final choice; development, test and implementation of highly efficient broadband quasi-optical mode converters, diplexers (FADIS), polarizers, directional couplers; FADIS switching of $0.5 \mathrm{MW}$ at $25 \mathrm{kHz}$; successful $0.9 \mathrm{MW}$, $30 \mathrm{~min}$. demonstration of the quasi-optical multibeam transmission line of W7-X; development and successful high-power test of the remote-steering launchers at W7-X; fast synthesis of wideband multimode waveguide components; development, test and implementation of corrugated, high-mode purity HE 11 -hybrid mode transmission line components and dummy loads; integration of the ITER upper launcher.

\section{Non-Fusion Applications of Gyrotrons}

Application of highly-efficient gyrotrons in ECR heavy ion sources and in technological installations for microwave materials processing as sintering of advanced ceramics and metal-powder compacts as well as for microwave-assisted fabrication of low-loss, large-size CVD diamond disks. Studies on non-thermal microwave effects.

Development and application of frequency-stable $\mathrm{THz}$ gyrotrons in Dynamic Nuclear Polarization (DNP) spectroscopy Nuclear Magnetic Resonance (NMR) experiments, for plasma diagnostics and for detection of concealed radioactive materials.

Development of pulsed broadband gyro-TWTs and CW gyro-BWOs with helical interaction circuit for RADAR applications and materials processing. 